\title{
EFFECTS OF INFECTIONS ON SEVERELY MALNOURISHED CHILDREN IN KILIFI-MOMBASA AND DAR ES SALAAM: A COMPARATIVE STUDY.
}

\begin{abstract}
Objective: To determine the effects of infections on severely malnourished children.
\end{abstract}

Study design: Descriptive, cross-sectional hospital based study.

Results: There is little difference in the prevalence of severe malnutrition between the two hospitals. 55\% of all malnourished children were boys. Nonoedematous Protein Energy Malnutrition was more prevalent at Muhimbili National Hospital than at Kilifi District Hospital. More than $75 \%$ of all severe Protein Energy Malnutrition patients were below two years of age, $36 \%$ of all severe Protein Energy Malnutrition patients admitted also had malaria, $45 \%$ of all admitted patients with severe Protein Energy Malnutrition at Kilifi District Hospital also had diarrhoea. More than $25 \%$ of severely malnourished patients died, oedematous type having a higher case fatality rate than non-oedematous one. $86 \%$ of the patients who died at MNH had other co-morbidities.

Septicaemic malnourished children succumbed more deaths than other co infections.

Conlusion: Infections cause most deaths and complicate the management of severe malnutrition. Severe malnutrition is still a big problem among these countries. 


\section{INTRODUCTION}

Human life, growth and well-being need nutritional substances. In children demand of these substances varies with age, sex and development. Excess or deficiency of these nutrients, leads to a condition called malnutrition, which is classified into obesity and under nutrition respectvely'. Malnutrition is a factor in $60 \%$ of the 11 million deaths that occur each year in the world's 0-4 year old children ${ }^{2}$. The majority of world's children live in developing countries; lack of food and clean water, poor sanitation, infections and social unrest leads to LBW(Low Birth weight) and PEM (Protein Energy Malnutrition)

PEM is more common in developing countries among under fives, the severe form being between 1-10\% and underweight between 20-40\%. In the year 2000, WHO estimated that $32 \%$ of under fives in developing countries were underweight, $78 \%$ of these live in South East Asia and 15\% in Sub Saharan Africa.'

Severe malnutrition is defined as weight for height z-score <-3SD, bilateral oedema of kwashiorkor, or mid-upper arm circumference MUAC $<11.0 \mathrm{~cm}$ (if $>65 \mathrm{~cm}$ in length) ${ }^{3}$ PEM was clinically classified through the Wellcome trust classification based on Weight for Age (WFA) with or without oedema into the following grades; $80-60 \%$ without oedema as underweight., $80-60 \%$ with oedemaKwashiorkor; $<60 \%$ with oedema- Marasmickwashiorkor; $<60 \%$ without oedema as Marasmus.'

Kwashiorkor occurs in infancy but maximally in the $2^{\text {nd }}$ year following abrupt weaning, it is not only of nutritional origin, infections, psychosocial, and cultural factors are also operative. Clinically, the degree, duration, speed, age of onset, presence of conditioning factors and genetic factors affect it. Constant features include; oedema, psychomotor changes, growth retardation and muscle wasting. Usual signs include; moon face, hair changes, skin depigmentation and anaemia. Occasional presentation includes; hepatomegaly, flank paint dermatosis, cardiomyopathy, dehydration, signs of infection and micronutrient deficiency.

Marasmus generally means wasting, it involves inadequate intake of protein and calories, representing the end result of starvation. It occurs in the $1^{\text {st }}$ year of life due to lack of breastfeeding and use of diluted animal milk. Poverty and famine are usually precipitating factors while ignorance and poor maternal nutrition are also contributory. Clinical features include; severe wasting of muscles and subcutaneous fat, severe growth retardation, child looks older than his age, no oedema or hair changes, alert and miserable, hungry, diarrhoea and dehydration. ${ }^{1}$

Pre-school aged children in developing countries are often at risk of malnutrition because of their dependency on others for food, increased protein and energy requirements, and immature immune system causing greater susceptibility to infections. Prognosis is worse when PEM occurs with HIV infections. GIT (Gastro Intestinal Tract) infections precipitate PEM due to association with diarrhoea, anorexia, vomiting and increased metabolic need. Parasitic infections play a major role in many parts of the world. ${ }^{4}$

Severe malnutrition affects 1-2\% of pre-school children mainly in the developing countries. In Tanzania, about 5\% of all under fives are affected, marasmus being commoner than kwashiorkor in younger ages. Infections are inseparable to malnutrition affecting younger

DMSJ Vol. 14 (Suppl. 1) 
ages with an increased mortality and morbidity than children of normal nutrition status ${ }^{5}$.

InKenya malnutritionisincreasing in prevalence and is a serious public health problem ${ }^{6}$; a survey of 6419 children in 14 districts found that $27 \%$ of children were underweight and $6 \%$ were wasted ${ }^{7}$. In Kilifi District Hospital $(\mathrm{KDH}) 41 \%$ of children were underweight (weight for age percentile 60-80\%) and 16\% were severely malnourished (weight for age $<60 \%)^{3}$ Malnutrition is the fourth commonest cause of admission to $\mathrm{KDH}$

Malnutrition and infections are interrelated. Severe malnutrition leads to an immunodeficiency state known as NAIDs (Nutritionally Acquired Immune Dysfunctions) $)^{5}$ The ability of malnourished child to handle infections is lower, common infections being Streptococcus pneumoniae, Klebsiella pneumonia, Escherichia coli and Hemophilus influenza ${ }^{8}$. Higher incidence of urinary tract infections is also documented in severely malnourished children?. Common bacterial infections like pneumonia, septicaemia, diarrhoea, meningitis, tuberculosis and nosocomial infections are found in children with severe PEM in paediatric wards at Muhimbili National Hospital, MNH ${ }^{10}$.

The World Health Organisation (WHO) has defined standard criteria for the recognition and management of severe malnutrition in children and implementation of these guidelines ${ }^{11}$ has been shown to improve outcome ${ }^{6}$.

\section{METHODOLOGY}

Study design: This was a retrospective study, which included all malnourished patients admitted for the period of one year Study area: Paediatric wards at Kilifi District hospital, Kilfi and paediatric wards at MNH Kilifi is a district on the coast of Kenya situated $60 \mathrm{~km}$ north of Mombasa.The district hospital serves a population of over 230,000 and the paediatric unit admits more than 5000 children per year. Majority of the residents are subsistent farmers with only $4 \%$ estimated to have waged employment. The area is hyperendemic for malaria with two peaks during the year, April-June and November. Malnutrition is also endemic in the area with $41 \%$ of undernourished (weight for age percentile $60-80 \%)$ and $16 \%$ of severely malnourished (weight for age $<60 \%$ ) children.

MNH is Tanzania's national hospital, which also serves as the referral hospital for the Eastern zone., It is situated in Ilala district of Dar es salaam, acting as the refferal point for district hospitals in the city, moreover it receives referred patients from all the regions in the country.

Study population: All malnourished children admitted at these health facilities in one-year were included.

Sample size: A total of 781 severely malnourished patients who were admitted at $\mathrm{MNH}$ in one year and 341 patients admitted at $\mathrm{KDH}$ in one year were included

Data collection: Information from hospital register books, information concerning infections were obtained from patients' personal files, investigation forms and discharge or death summaries, at $\mathrm{MNH}$, and data from online system at KDH were used.

Data analysis: Data were analysed through STATA programme and epi info programme.

Inclusion criteria: All malnourished children admitted to these hospitals

Study limitations: There were difficulties in data collection at $\mathrm{MNH}$, since some basic information were missing from the admission records, however a lot was done to ensure that the available information was obtained and properly used. 


\section{RESULTS}

There were 1121 patients admitted in MNH and KDH with severe malnutrition for the duration of one year, the total of 616 were boys and girls

Table 1 sex distribution table

\begin{tabular}{|c|c|c|c|c|c|c|}
\hline \multicolumn{7}{|c|}{ sex distribution table } \\
\hline & $\mathrm{MNH}$ & & & $\mathrm{KDH}$ & & \\
\hline type & male & female & n & male & female & $n$ \\
\hline kwsh/MK & $156(56 \%)$ & $121(44 \%)$ & 277 & $106(51 \%)$ & $104(49 \%)$ & 210 \\
\hline Marasmus & $278(55 \%)$ & $226(45 \%)$ & 504 & $76(59 \%)$ & $54(41 \%)$ & 130 \\
\hline Total & $434(56 \%)$ & $347(44 \%)$ & 781 & $182(54 \%)$ & $158(46 \%)$ & 340 \\
\hline
\end{tabular}

About 55\% of the patients admitted with severe malnutrition in both hospitals were male patients; more female patients had severe oedematous malnutrition in $\mathrm{KDH}$ than in $\mathrm{MNH}$.

Table 2 Age group distribution of severely malnourished children

\begin{tabular}{|l|l|l|l|l|l|l|l|l|l|l|}
\hline \multicolumn{2}{|c|}{ MNH } & \multicolumn{1}{l|}{ KDH } & & & \\
Type & $<1 \mathrm{yr}$ & $1-2 \mathrm{yr}$ & $3-4 \mathrm{yr}$ & $>4 \mathrm{yr}$ & $\mathrm{n}$ & $<1 \mathrm{yr}$ & $1-2 \mathrm{yr}$ & $3-4 \mathrm{yr}$ & $>4 \mathrm{yr}$ & $\mathrm{n}$ \\
\hline Kwsh/MK & $36(13 \%)$ & $135(49 \%)$ & $60(22 \%)$ & $46(16 \%)$ & 277 & $10(8 \%)$ & $86(66 \%)$ & $29(22 \%)$ & $6(5 \%)$ & 131 \\
\hline Marasmus & $133(26 \%)$ & $272(54 \%)$ & $60(12 \%)$ & $39(8 \%)$ & 504 & $63(30 \%)$ & $109(52 \%)$ & $25(12 \%)$ & $13(6 \%)$ & 210 \\
\hline Total & $169(22 \%)$ & $407(52 \%)$ & $120(15 \%)$ & $85(11 \%)$ & 781 & $73(21 \%)$ & $195(57.2 \%)$ & $54(16 \%)$ & $19(6 \%)$ & 341 \\
\hline
\end{tabular}

About $54 \%$ of all severely malnourished patients were within the age interval 1-2 years, under one year children admitted had the same prevalence of severe malnutrition in both hospitals.

Table3 MORBIDITY Infections among severely malnourished patients

\begin{tabular}{|l|r|r|r|r|r|r|}
\hline \multirow{2}{*}{ Infections } & \multicolumn{5}{|l|}{ MNH } & \multicolumn{1}{l|}{ KDH } \\
\cline { 2 - 7 } Kwsh/MK & Marasmus & \multicolumn{1}{l}{ Total } & \multicolumn{1}{l|}{ Kwsh/MK } & Marasmus & \multicolumn{1}{l|}{ Total } \\
\hline Malaria & $99(24 \%)$ & $227(22 \%)$ & $326(21 \%)$ & $49(20 \%)$ & $29(15 \%)$ & $78(18 \%)$ \\
\hline PAIDS & $68(16 \%)$ & $221(22 \%)$ & $289(19 \%)$ & $8(3 \%)$ & $30(16 \%)$ & $38(9 \%)$ \\
\hline Diarrhoea & $34(8 \%)$ & $82(8 \%)$ & $116(8 \%)$ & $122(51 \%)$ & $73(38 \%)$ & $195(45 \%)$ \\
\hline LRTI & $75(18 \%)$ & $200(20 \%)$ & $275(18 \%)$ & $10(42 \%)$ & $19(10 \%)$ & $29(7 \%)$ \\
\hline TB & $57(14 \%)$ & $119(12 \%)$ & $276(18 \%)$ & $4(2 \%)$ & $13(7 \%)$ & $17(4 \%)$ \\
\hline UTI & $37(9 \%)$ & $84(8 \%)$ & $121(8 \%)$ & & & \\
\hline Septicaemia & $25(6 \%)$ & $18(2 \%)$ & $43(3 \%)$ & $9(4 \%)$ & $11(6 \%)$ & $20(5 \%)$ \\
\hline Candidiasis & $12(3 \%)$ & $56(6 \%)$ & $68(4 \%)$ & $37(15 \%)$ & $14(7 \%)$ & $51(12 \%)$ \\
\hline OM & $10(2 \%)$ & $17(2 \%)$ & $27(2 \%)$ & $2(1 \%)$ & $1(1 \%)$ & $3(1 \%)$ \\
\hline Meningitis & & & & 0 & $2(1 \%)$ & $2(1 \%)$ \\
\hline N & 417 & 1024 & 1541 & 241 & 192 & 435 \\
\hline
\end{tabular}

DMSJ Vol. 14 (Suppl. 1) 
Malaria was the leading co morbidity with $45 \%$ of all admitted severely malnourished patients followed by HIV and diarrhoea. There were also patients with mixed co morbidities. Diarrhoea was the most common co morbidity at $\mathrm{KDH}$ than at $\mathrm{MNH}$ with more than half oedematous malnourished children at KDH having it. Both HIV and malaria were more prevalent at MNH than at KDH.

\section{Table 4 MORTALITY}

\begin{tabular}{|l|l|l|l|l|l|l|l|}
\hline \multirow{3}{*}{ Type } & MNH & KDH & & \\
\cline { 2 - 8 } & Death & Disch & N & Death & Disch & Abscond & N \\
\hline Kwsh/MK & $121(44 \%)$ & $156(56 \%)$ & 277 & $30(23 \%)$ & $92(70 \%)$ & $9(7 \%)$ & 131 \\
\hline Marasmus & $101(20 \%)$ & $403(80 \%)$ & 504 & $34(16 \%)$ & $173(82 \%)$ & $3(1 \%)$ & 210 \\
\hline Total & $222(28 \%)$ & $559(72 \%)$ & 781 & $64(19 \%)$ & $265(78 \%)$ & $12(4 \%)$ & 341 \\
\hline
\end{tabular}

More than a quarter of severely malnourished children died, however there were more mortality noted at MNH than at $\mathrm{KDH}$. Oedematous type of severely malnourished children had more case fatality rates than non-oedematous

\section{Table 5 Outcome in severe malnourished patients with infections}

\begin{tabular}{|l|r|r|r|r|}
\hline & \multicolumn{3}{|l|}{ KDH } & \\
\cline { 2 - 5 } Co morbidity & Death & Discharged & Abscond & \multicolumn{1}{c|}{$\mathrm{n}$} \\
Malaria & $7(9 \%)$ & $67(86 \%)$ & $3(4 \%)$ & 78 \\
\hline PAIDS & $9(24 \%)$ & $27(71 \%)$ & $2(5 \%)$ & 38 \\
\hline Diarrhoea & $40(21 \%)$ & $145(74 \%)$ & $10(5 \%)$ & 195 \\
\hline LRTI & $6(21 \%)$ & $23(79 \%)$ & & 29 \\
\hline TB & $4(24 \%)$ & $13(76 \%)$ & & 17 \\
\hline Septicaemia & $11(55 \%)$ & $8(40 \%)$ & $1(5 \%)$ & 20 \\
\hline Candidiasis & $44(15 \%)$ & $234(81 \%)$ & $10(3 \%)$ & 290 \\
\hline Otitis media & & $3(100 \%)$ & & 3 \\
\hline Meningitis & $1(50 \%)$ & $1(50 \%)$ & & 2 \\
\hline Total & $122(18 \%)$ & $521(76 \%)$ & $26(4 \%)$ & 672 \\
\hline
\end{tabular}

At MNH, of the 222 patients who died, $86 \%$ had co morbidities. More deaths were noted in patients with oedematous malnutrition and co morbidities than none oedematous type. About $46 \%$ of oedematous patients with co-infections died compared to only $19 \%$ of non-oedematous patients with co-infections. Data of deaths caused by specific infections at MNH were not available for analysis. At KDH, septicaemia was the most common cause of death followed by TB and HIV

\section{Trends}

Prevalence of severe malnutrition increases with age up to the maximum at the age interval 12years, there after it decreases as children gets older. Age group vs. malnutrition distribution showed 
similar prevalence in both hospital among under ones, and about $75 \%$ of all severe malnourished children were below 2 years.

Trends of mortality with age-KDH

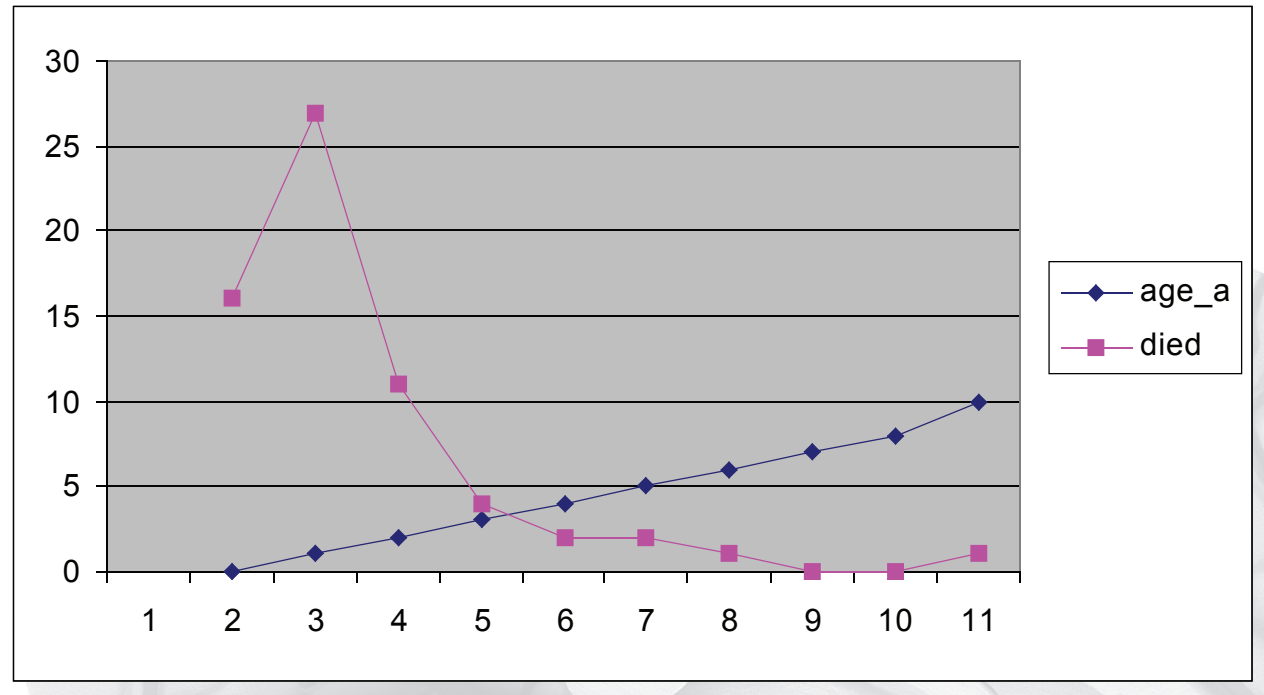

Mortality rates in $\mathrm{KDH}$ increase with age up to the maximum deaths of $42 \%$ and then decrease after the $2^{\text {nd }}$ year of life.

The numbers of the patients tend to increaseas the severity of malnutrition increases i.e. $35 \%$ with oedematous malnutrition to $62 \%$ of marasmus. This trend is reverse with $\mathrm{KDH}$ where the trend of prevalence decreases with the severity from $63 \%$ of oedematous to $38 \%$ of non-oedematous malnutrition.

\section{DISCUSSION}

This descriptive comparative study between $\mathrm{MNH}$ and $\mathrm{KDH}$ for one year admissions of severely malnourished children revealed the prevalence of $13.6 \%$ in $\mathrm{MNH}$. However the prevalence of this condition among the total admission in $\mathrm{KDH}$ was $16 \%{ }^{3}$. The prevalence is almost similar in these two hospitals, the small difference could be attributed by the lack of online data storage system at MNH as compared to that of $\mathrm{KDH}$ thus leading to loss of some information.

Marasmus was found to be more prevalent in $\mathrm{MNH}{ }^{5} \mathrm{Compared}$ to $\mathrm{KDH}$; conversely severe form of oedematous malnutrition is more prevalent at $\mathrm{KDH}$ than at $\mathrm{MNH}$. It is also found out that boys succumbs more incidences of severe malnutrition than girls in both hospitals.

More than half of the severely malnourished patients were in the age interval 1-2years. This is may be because this is the time that breast feeding is stopped and the staple foods are fully taking over, these foods may not contain all required nutrients supply especially protein and calories '. It is also at this age that children pick a lot of infectious objects from the soil and eats them facilitating STH(Soil Transmitted Helminthes) transmission and other GIT infections, however the trend of the prevalence decreases as the child gets older ${ }^{1,4}$ . Both kwashiorkor and marasmus peak at the age of 2years due to the factors like weaning, infections, cultural and psychological, 1

DMSJ Vol. 14 (Suppl. 1) 
there has been no significant differences in prevalence between the two hospitals.

Children with severe malnutrition are more prone to infections than others. This is due to the condition called NAIDS (nutritionally acquired immune dysfunctions syndrome) in which a child becomes immunocompromised and suffers bacterial, fungal and other infections. ${ }^{4,5}$ Ability of the malnourished child to handle infections is lower ${ }^{8} \mathrm{In} \mathrm{MNH}$ about $89 \%$ of patients with severe form of malnutrition had co morbidities, malaria is the leading co morbidity followed by HIV and diarrhoea. Malaria, although it is an acute febrile illness causes malnutrition when the child suffers recurrent infections leading to loss of appetite, nausea and vomiting, which limits the quantity of food that a child will take hence leading to higher mortality and morbidity ${ }^{14}$ HIV causes immunosuppression, it causes mixed infections and hence exacerbates malnutrition ${ }^{8}$. (Lower Respiratory Tract Infections) LRTI, TB and septicaemia are also prevalent in malnourished patients ${ }^{8}$. Diarrhoea is the leading co-morbidity at $\mathrm{KDH}$ where in $\mathrm{MNH}$, malaria is the leading co morbidity, this may be due to the fact that in Kilifi district the socio-economic status is lower than Dar es Salaam since diarrhoea is the disease of poverty. Diarrhoea causes malnutrition due to inadequate absorption and loss of nutrients and water.

$26 \%$ of all children admitted due to severe malnutrition died, mortality is observed more in $\mathrm{MNH}$ than $\mathrm{KDH}$. This may be due to strictly following of the WHO guidance of management of these conditions, with proper management; the mortality should be less than $5 \% .{ }^{12}$ Mortality is observed more in children with oedematous malnutrition than those with no oedema in both settings, again the case fatality rate being higher at MNH. The most common complications, which kill these patients, are hypoglycaemia, hypothermia, infections, dehydration and electrolyte imbalance. $^{2}$

Infections caused majority of deaths to these patients. In MNH 86\% of the patients who died had co morbidities. More deaths were noted in patients with oedematous type of malnutrition. Oedema among other complications it causes congestive heart failure and pulmonary oedema. ${ }^{6}$ At $\mathrm{KDH}$, more deaths were noted in patients with septicaemia, in which more than a half died, many deaths in severe malnutrition are attributed to bacteraemia among other factors. ${ }^{8} \mathrm{HIV}$, TB are the other many causes of death.

The trend of prevalence of severe malnutrition usually increases after the sixth month of life mostly due to weaning and introduction of other staple foods, it further increases up to its maximum at the age around two years, mainly because of lack of nutritious food and infections. ${ }^{1,5}$ more than $75 \%$ of all severe malnourished admissions were below 2years of age. Mortality also increases with age up to two years and then drops, as the child gets older. This is probably because of the high prevalence of the patients with this age.

\section{CONCLUSION}

There is a small difference of prevalence between $\mathrm{MNH}$ and $\mathrm{KDH}$. Both of them have high prevalence despite of the current WHO guidance for management of these conditions

Boys are more affected by severe malnutrition than girls in both hospitals.

Oedematous malnutrition is more common than non-oedematous type in $\mathrm{KDH}$, while non-oedematous type is more common than oedematous type at $\mathrm{MNH}$.

DMSJ Vol. 14 (Suppl. 1) 
The prevalence and case fatality rates are increasing with age up to the maximum in the second year of life and declining afterwards as the child gets older

Malaria is the leading co-morbidity among total admissions, however diarrhoea is the leading co-morbidity at $\mathrm{KDH}$ alone compared to malaria at $\mathrm{MNH}$.

Case fatality rate is higher among $\mathrm{MNH}$ admissions than those of $\mathrm{KDH}$. Oedematous malnourished patients had more deaths than non-oedematous.

Malnourished patients with co-morbidities had higher case fatality rate than those with no co-morbidities; patients who had septicaemia had the highest case fatality rate.

\section{RECOMMENDATION}

There should be modern data storing mechanism, like the one that is existing at $\mathrm{KDH}$, for better results. This will help as to see whether the situation is worsening or improving over time. This will also serve the long time that the researchers will use to obtain the data concerned.

Current WHO guidelines for management of severe malnutrition should be revised since the intended outcome of bringing the mortality rate down to $5 \%$ has not been the outcome so far.
The population should be encouraged to seek medical attention earlier during any childhood illness.

Nutrition workout should be considered for younger children especially after weaning period up to two years, young children should be dewormed frequently.

Proper management of malnourished children should be considered especially those with other co morbidities.

\section{AKNOWLEDGEMENT}

I would like to send my sincerely appreciation to my supervisors, Drs Sarah, James and Ndiritu for their time that they dedicated for me. Also, I would like to send my word of appreciation to Director of KEMRI/WELLCOMETRUST RESEARCH COLLABORATION PROGRAMME, Prof Kevin Marsh as well as the Director of clinical researches Prof. Charles Newton and the whole management team for facilitating my elective study at Kiliff for six weeks.

Last but not least I would like to send my sincere gratitude to my elective coordinator and the first supervisor Dr Julie Makani, for the support and assistance that she gave before and during my elective period. Lastly I would like to thank the management of MUCHS for all the support and the assistance they gave me.

DMSJ Vol. 14 (Suppl. 1) 


\section{REFERENCES}

1. Lecture notes by Abdelaziz Elamin, Prof of child health college of Medicine, Sultan Quabos University, Muscat, Oman.

2. WHO. Improving child health in the community. WHO/FCH/CAH/02.12. Geneva:WHO; 2002

3. Berkley JA. Invasive bacterial infections in children at a sub-Saharan District Hospital. MD Thesis. Newcastle: University of Newcastle upon Tyne; 2002

4. Andrew Lin, MD, Associate Prof, Dept of internal medicine, source e-medicine. com.

5. Isaac H. Pattern of bacterial infections among the Severely Malnourished Children in PediatricWards of Muhimbili Medical Center; Dissertation for MMed, University of Dar es Salaam, 1990

6. Ashworth A, Chopra M, McCoy D, Sanders D, Jackson D, Karaolis N, Sogaula N, Schofield C. WHO guidelines for management of severe malnutrition in rural South African hospitals: effect on case fatality and the influence of operational factors. Lancet. 2004; 363:1110-1115

7. Ngare DK, Muttunga JN. Prevalence of malnutrition in Kenya. East Afr Med J. 1999; 76:376-380

8. BerkowitzFE.Bacteremiainhospitalized black South African children. A oneyear study emphasizing nosocomial bacteremia and bacteremia in severely malnourished children. Am J Dis Child. 1984; 138:551-556

9. Philip I et al: BMJ 1988; Acute Bacterial Infections in Kwashiorkor and Marasmus.

10. Isaac H: East Africa Medical Journal 1992; Nosocomial Bacterial Infections Among Children with Severe PEM.

11. WHO. Management of Severe Malnutrition: a Manual for Physicians and other Senior Health Workers. Geneva; 1999 